\section{Double-channel colonoscopic snare resection of large pedunculated polyps using a clipping method}

The predominant complication associated with colonoscopic polypectomy is bleeding, the rate ranging from $0.44 \%$ to $2.6 \%$ [1,2]. Iida et al. [3] used a clipping method for endoscopic polypectomy in 40 patients. However, this maneuver might be quite difficult to accomplish with a single-channel endoscope when the endoscopic view of the pedicle is limited. We developed a new and easy clipping technique for double-channel colonoscopic snare resection of large pedunculated polyps in an attempt to reduce the incidence of hemorrhage.

The procedure was performed as follows. A double-channel colonoscope (CF2T200; Olympus Optical Co., Tokyo, Japan) was inserted to the level of the polyp. An Olympus SD 5U-1 snare was passed through the right channel and the wire loop was placed and closed over the stalk. The polyp head was tented away from the colon wall, providing adequate visualization of the stalk ( $\bullet$ Figure $1 \mathbf{a}$ ). An Olympus HX-600-135 clip was passed through the second channel and fitted onto the pedicle, near enough to the colon wall to allow transection of the stalk above the clip with a sufficiently large margin ( $\bullet$ Figure $\mathbf{1} \mathbf{b}$ ). It is important that the wire snare does not touch the metal clip in case an aberrant current pathway is activated which could burn the colon wall. The stalk was then transected using electrocautery and snare closure ( $\bullet$ Figure $1 \mathrm{c})$.

We treated a total of 15 pedunculated colorectal polyps (in 12 patients) with heads all measuring over $10 \mathrm{~mm}$ in diameter (mean $20 \mathrm{~mm}$, range $11-32 \mathrm{~mm}$ ). All the lesions were easily and safely resected. The clipping technique itself took an average of 4 minutes (range 3-6 minutes). No massive bleeding and no perforations occurred during or after polypectomy, and there were no clip-related complications. These preliminary results suggest that our method is an easy and reliable technique for the prevention of postpolypectomy hemorrhage.
M. H. Tsai ${ }^{1,2}$, K. Wang ${ }^{2,3}$, S. J. Chou ${ }^{1,2}$, V. Chen ${ }^{1,2}$, Y. C. Yang ${ }^{4}$

1 Department of Surgery, Cardinal Tien Hospital, Taipei, Taiwan

2 School of Medicine, Fu-jen Catholic University, Taipei, Taiwan

3 Department of Internal Medicine, Cardinal Tien Hospital, Taipei, Taiwan

4 Department of Clinical Laboratory Sciences and Medical Biotechnology, College of Medicine, National Taiwan University, Taipei, Taiwan

\section{References}

1 Muhldorfer SM, Kekos G, Hahn EG et al. Complications of therapeutic gastrointestinal endoscopy. Endoscopy 1992; 24: 276-283

2 Van Gossum A, Cozzoli A, Adler $M$ et al. Colonoscopic snare polypectomy: analysis of 1485 resections comparing two types of current. Gastrointest Endosc 1992; 38: $472-475$

3 Iida $Y$, Miura S, Munemoto $Y$ et al. Endoscopic resection of large colorectal polyps using a clipping method. Dis Colon Rectum 1994; 37: 179- 180

Bibliography

DOI 10.1055/s-2007-966409

Endoscopy 2007; 39: E200

(c) Georg Thieme Verlag KG Stuttgart · New York . ISSN 0013-726X

\section{Corresponding author}

\section{Y. C. Yang, PhD}

Department of Clinical Laboratory Sciences and Medical Biotechnology

College of Medicine

National Taiwan University

No. 1 Chang-Te Street

Taipei 100

Taiwan

Fax: +886-2-23711574

ycyang@ha.mc.ntu.edu.tw
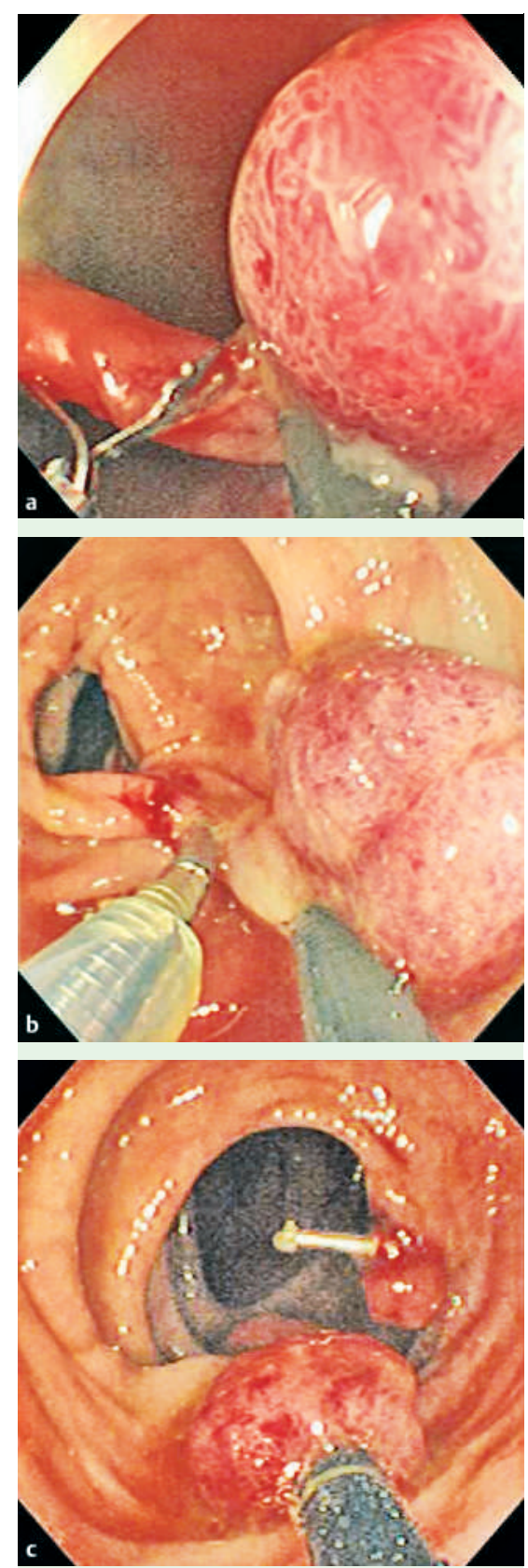

Figure 1 Endoscopic views showing the clipping technique for double-channel colonoscopic snare resection of large pedunculated polyps. a The snare loop can be seen completely encircling a pedunculated polyp. The polyp head is tented away from the colon wall, providing adequate visualization of the stalk. A clip has been passed through the second instrument channel to the level of the pedicle. $\mathbf{b}$ The clip is fitted onto the pedicle near enough to the colon wall to allow transection of the stalk above the clip with an adequate margin. c The stalk is transected, leaving the clip in place with no bleeding at the excision site. The polyp is grasped with a tripod. 